\title{
Comparative genomic analysis of Acinetobacter strains isolated from murine colonic crypts
}

\author{
Azadeh Saffarian', Marie Touchon², Céline Mulet ${ }^{1}$, Régis Tournebize ${ }^{3}$, Virginie Passet ${ }^{2}$, Sylvain Brisse², \\ Eduardo P. C. Rocha ${ }^{2}$, Philippe J. Sansonetti ${ }^{1,4}$ and Thierry Pédron ${ }^{1 *}$ (i)
}

\begin{abstract}
Background: A restricted set of aerobic bacteria dominated by the Acinetobacter genus was identified in murine intestinal colonic crypts. The vicinity of such bacteria with intestinal stem cells could indicate that they protect the crypt against cytotoxic and genotoxic signals. Genome analyses of these bacteria were performed to better appreciate their biodegradative capacities.

Results: Two taxonomically different clusters of Acinetobacter were isolated from murine proximal colonic crypts, one was identified as $A$. modestus and the other as A. radioresistens. Their identification was performed through biochemical parameters and housekeeping gene sequencing. After selection of one strain of each cluster (A. modestus CM11G and A. radioresistens CM38.2), comparative genomic analysis was performed on whole-genome sequencing data. The antibiotic resistance pattern of these two strains is different, in line with the many genes involved in resistance to heavy metals identified in both genomes. Moreover whereas the operon benABCDE involved in benzoate metabolism is encoded by the two genomes, the operon ant $A B C$ encoding the anthranilate dioxygenase, and the phenol hydroxylase gene cluster are absent in the A. modestus genomic sequence, indicating that the two strains have different capacities to metabolize xenobiotics. A common feature of the two strains is the presence of a type IV pili system, and the presence of genes encoding proteins pertaining to secretion systems such as Type I and Type II secretion systems.
\end{abstract}

Conclusions: Our comparative genomic analysis revealed that different Acinetobacter isolated from the same biological niche, even if they share a large majority of genes, possess unique features that could play a specific role in the protection of the intestinal crypt.

Keywords: Acinetobacter, Murine intestine, Comparative genomics, Xenobiotics

\section{Background}

Acinetobacter, belonging to $\gamma$-proteobacteria, are gramnegative strictly aerobic, non-motile, non-fermentative and oxidase-negative bacteria. The classification of the genus Acinetobacter contains over 50 species [1]. Acinetobacter spp. were considered for decades as saprophytic environmental microorganisms. However, recently, they have increasingly been implicated in various types of infections, mainly nosocomial infections in fragilized

\footnotetext{
* Correspondence: thierry.pedron@pasteur.fr

'Unité de Pathogénie Microbienne Moléculaire, INSERM U1202, Institut

Pasteur, Paris, France

Full list of author information is available at the end of the article
}

patients in intensive care units, hence adding the hospital to the list of their favorite environments. A major trait of their pathogenicity is their high and broad array of antibiotic resistance. The often multi-drug resistance (MDR) Acinetobacter baumannii is the major species in the genus involved in recent nosocomial infections. In contrast to A. baumannii that is mainly found in the hospital environment, other species of the Acinetobacter genus are isolated from the soil, water, and animals [2]. Many environmental Acinetobacter spp. are able to metabolize pollutants such as "Acinetobacter oleivorans" DR1 that degrades diesel [3] and the strains Acinetobacter 
pittii PHEA-2 and Acinetobacter baylyi ADP1 that degrade phenol [4].

Our previous data showed the existence of a Crypt Specific Core Microbiota (CSCM) in the caecum and proximal colon of laboratory mice of various lineages, and identified a restricted set of strictly aerobic, nonfermentative bacterial genus, dominated by members of the Acinetobacter genus showing unexpected tropism for the crypt environment [5]. It was shown that oxygen is present at the gut mucosal surface at low but significant concentration thereby facilitating the growth of strictly aerobic and aero-anaerobic bacteria, allowing them to be biochemically active [6]. We hypothesize that these bacteria act as a crypt "gate keeper" by protecting the crypt regenerative apparatus, particularly stem cells, against cytotoxic and genotoxic signals. This may occur by several means: by preventing colonization by pathobionts, by regulating local innate immune mechanisms to avoid chronic low grade inflammation, and by providing strong biodegradative capacities against xenobiotics leading to putative cancer protective effects. Indeed as these bacteria are in close vicinity of intestinal stem cells we hypothesize that they act in order to keep homeostasis in this particular niche and also participate to the control of intestinal proliferation. The aim of the present study was to characterize and compare the genome of two different strains of Acinetobacter isolated from murine colonic crypts. We show here that these two strains belong to two different species of Acinetobacter found in the crypts (Acinetobacter modestus and Acinetobacter radioresistens), harbor different patterns of antibiotic resistance and also possess different xenobiotic degradative properties. It is interesting to notice, for instance, that efficient dehalogenation of xenobiotics requires aerobic conditions and that the CSCM are aerobic bacteria.

\section{Methods}

Isolation of crypt specific core Acinetobacter from murine proximal colon

Proximal colonic tissues from $\mathrm{C} 57 \mathrm{Bl} / 6$ mice (Elevage Janvier) were washed with bleach and homogenized using in $2 \mathrm{ml}$ of sterile PBS using the Precellys system with $2,8 \mathrm{~mm}$ ceramic beads and added to $30 \mathrm{ml}$ of a minimum medium [7]. The cultures were incubated at $30{ }^{\circ} \mathrm{C}$ during $48 \mathrm{H}$ under shaking condition (300 rpm). The cultures were then isolated on agar plates (GTCS, MacConkey, Herellea, ChromAgar). Selected colonies were then re-isolated on Chromagar plates. Bacteria were identified using the Biolog system (GEN III MicroPlate for both Gram-negative \& Gram-positive bacteria, 21,124 Cabot Blvd. Hayward CA, 94,545 USA). The identification of Acinetobacter was confirmed by Sanger sequencing of $16 S \mathrm{rDNA}$, and recombinase A (recA) after genomic DNA extraction using the Wizard Genomic
DNA Purification Kit following manufacture's instructions (Promega) and PCR amplification. The primers used are listed in the Additional file 1: Table S1.

\section{Antimicrobial susceptibility testing}

Antibiotic susceptibility was determined by the disk diffusion method on Mueller-Hinton ( $\mathrm{MH}$ ) agar according to the guidelines of the Antibiogram Committee of the French Microbiology Society [8]. Automatic readings were performed using the OSIRIS system (Bio-Rad).

\section{Biofilm assay}

Biofilm formation was determined using an overnight culture, diluted 1:100 in fresh Trypticase-Soy broth in 96-well polystyrene plates and incubated without shaking at $37{ }^{\circ} \mathrm{C}$ for $24 \mathrm{~h}$ and $48 \mathrm{~h}$. After incubation the plates were washed gently three times with phosphatebuffered saline to remove unattached bacteria, air-dried and stained with $0.1 \%$ crystal violet solution for $20 \mathrm{~min}$ and quantified at $595 \mathrm{~nm}$ after solubilization with ethanol-acetone [9]. Of the 96 wells, six were left uninoculated and used as background controls. Escherichia coli DH5 $\alpha$ and Acinetobacter baumannii CIP $70.34^{\mathrm{T}}$ (ATCC 19606) were used respectively as negative and positive control of biofilm formation [10]. The biofilm formation experiments were carried out with six replicates and the results are expressed as mean values of crystal violet absorbance \pm SD from the mean.

\section{Genome sequencing, assembling, annotation}

The genome sequencing, assembling and annotations of A. modestus CM11G and A. radioresistens CM38.2 obtained through Illumina paired-end sequencing were already described in [11]. The complete genome sequence of $A$. radioresistens CM38.2 was obtained using PacBio single-molecule real-time (SMRT) technology [12] with P6-C4 chemistry in the PacBio RS II sequencing platform (https://www.gatc-biotech.com). One SMRT cell generated 72,134 reads with a mean read length of $15,211 \mathrm{bp}$. The reads were assembled de novo with the Hierarchical Genome Assembly Process 3 (HGAP3) [13] giving one contig of 3,201,807 bp with a G + C \% of 41.7 and with an average coverage depth of 275 . Whole-genome alignment of the A. radioresistens CM38.2 strain was performed using Mauve v 2.31 software [14] in order to compare the sequences obtained either by PacBio or by Illumina paired-end sequencing (Additional file 2: Figure S1).

The complete sequence of CM38.2 and all 118 contigs described in [11] of CM11G were annotated using the RAST and MicroScope platforms [15-17]. For both platforms our annotation job were submitted by providing mandatory information and accepting default parameters. Both platforms give access to several tools of visualization and comparative genomics and produced 
very similar results. In order to homogenize the results, we used only the annotations obtained with RAST.

\section{Core-genomes}

The core-genome of the species is defined as the intersection of pairwise lists of strict positional orthologs. We built three core-genomes: i) containing the 133 Acinetobacter strains used in [18] plus our two strains CM11G, CM38.2, ii) for the species $A$. radioresistens, iii) for $A$. modestus. All three core-genomes were built following the same protocol (as detailed in [19]). Briefly, orthologs were identified as bidirectional best hits using end-gap free global alignment, between the proteome of $A$. baumannii AYE as a pivot and each of the other proteomes (135 for the genus and 3 for the two species). Hits with less than $40 \%$ (genus) or $80 \%$ (species) similarity in amino acid sequence or more than $20 \%$ difference in protein length were discarded. Genomes from the same species typically show low levels of genome rearrangements and this information can be used to identify orthologs more accurately $[20,21]$. The core-genomes consist in the genes present in all genomes of each of the three sets.

Average nucleotide identity (ANIb) was computed using JSpecies v.1.2.1 [http://imedea.uib-csic.es/jspecies/] based on BLAST+ (v.2.2.29). The ANIb values were thus generated, on the one hand for pair-wise comparisons of CM38.2 and $A$. radioresistens strains NIPH 2130 (accession number NZ_APQE00000000.1) and CIP 103788 (accession number NZ_APQF00000000.1), and on the other hand for the comparison of CM11G with A. modestus strains ANC 3862 (accession number NZ_APRP00000000.1) and NIPH 236 (accession number NZ_APOJ00000000.1).

\section{Pan-genomes}

The pan-genome of the species is defined as the union of all the homologs present in the genes set of all strains, and provides information regarding the genetic diversity of the set of genomes. The pan-genomes were built for the same three sets of strains previously described (see Core-genomes section). In each case, the pan-genome was obtained by clustering homologous proteins into families. The lists of putative homologs between pairs of genomes using "all-against-all" comparisons with BLASTp v.2.2.28+ (default parameters) [22] were determined and then clustered by similarity using Silix v1.2.8 (http://lbbe.univ-lyon1.fr/SiLiX) [23] when the e-values were smaller than $10^{-4}$. A protein is thus included in the family if it shares a relation of homology to a protein already in the family. Silix parameters were set such that a protein was homologous to another in a given family if the alignment had at least $40 \%$ (genus) or $80 \%$ (species) sequence identity and included more than $80 \%$ of the smallest protein.

\section{Phylogenetic analyses}

For each of the phylogenetic reconstructions, we used the model minimizing the Bayesian Information Criterion (BIC) among all models available (option -m TEST) in IQ-TREE. We made 1000 ultra fast bootstraps to evaluate node support (options -bb 1000 -wbtl in IQ-TREE).

\section{5 rRNA gene phylogenetic tree}

We built a tree to display the phylogenetic distribution of our dataset using the 16S rRNA genes sequences of the 133 Acinetobacter strains used in [18] plus our 10 additional strains. We made a multiple alignment of the 16S rRNA sequences with INFERNAL v.1.1 (default parameters) [24] using RF00177 Rfam model (v.12.1, [25]), followed by manual correction with SEAVIEW to remove poorly aligned regions. The tree was computed by maximum likelihood with IQ-TREE multicore v.1.4.2 [26] under the TVM + I + G4 model.

\section{recA gene phylogenetic tree}

We extracted from the genus core-genome the gene family encoding RecA. Then we made multiple alignment of the set including the 135 recA DNA sequences and our eight additional strains with MAFFT v.7.205 (default parameters) [27], followed by manual correction with SEAVIEW to remove poorly aligned regions. The phylogenetic tree was inferred using IQ-TREE multicore v.1.4.2 under the TIM3 + I + G4 model.

\section{Core-genome phylogenetic tree}

Each of the 945 families of proteins of the Acinetobacter core-genome was used to produce a multiple alignment with MAFFT v.7.205 (default parameters) [27]. Poorly aligned regions were removed with BMGE (default parameters) [28]. The phylogenetic tree was inferred using IQ-TREE multicore v.1.4.2 under the LG + I + G4 + F model.

\section{Results}

Isolation and characterization of Acinetobacter strains in murine colonic crypts

As Acinetobacter was the major genus found in murine proximal colonic crypts, a selective minimum medium was used in order to isolate these strains $[7,11]$. Among the different preparations, ten Acinetobacter strains were isolated on ChromAgar plates and identified using the Biolog System based on 71 carbon source utilization assays and 23 chemical sensitivity assays. Eight of the strains were identified as Acinetobacter genospecies 6 and the two others as $A$. radioresistens indicating a clear separation of the ten strains into two clusters (Table 1). 
Table 1 Biochemical identification of the isolated strains

\begin{tabular}{ll}
\hline Strain & Biolog Identification \\
\hline CM11G & Acinetobacter genospecies 6 \\
CM31.3 & Acinetobacter genospecies 6 \\
CM31.5 & Acinetobacter genospecies 6 \\
CM31.6 & Acinetobacter genospecies 6 \\
CM32.1_HC & Acinetobacter genospecies 6 \\
CM32.1 & Acinetobacter genospecies 6 \\
CM37.1 & Acinetobacter genospecies 6 \\
CM37.2 & Acinetobacter genospecies 6 \\
CM38.1 & Acinetobacter radioresistens \\
CM38.2 & Acinetobacter radioresistens
\end{tabular}

10 Acinetobacter isolates were identified by the Biolog System using GEN III MicroPlate based on 71 carbon source utilization assays and 23 chemical sensitivity assays
Phylogeny of $16 \mathrm{~S}$ ribosomal RNA and recombinase a genes In order to get a more precise identification of the strains, a phylogenetic tree based on $16 \mathrm{~S}$ ribosomal RNA sequences was built. This phylogenetic tree confirmed the biochemical identification of strains CM38.1 and CM38.2 as closely related to $A$. radioresistens, and indicated that the eight other strains previously identified as Acinetobacter genospecies 6 were closely related to $A$. modestus species [29] (Additional file 3: Figure S2). In order to confirm these results we built a phylogenetic tree based on the $r e c A$ sequences because this gene was used to identify the different genospecies of the genus Acinetobacter [30]. This tree positioned these eight strains in the vicinity of the $A$. modestus species with more than $99 \%$ of sequence similarity and the two strains CM38.1 and CM38.2 in the vicinity of $A$. radioresistens (Fig. 1).

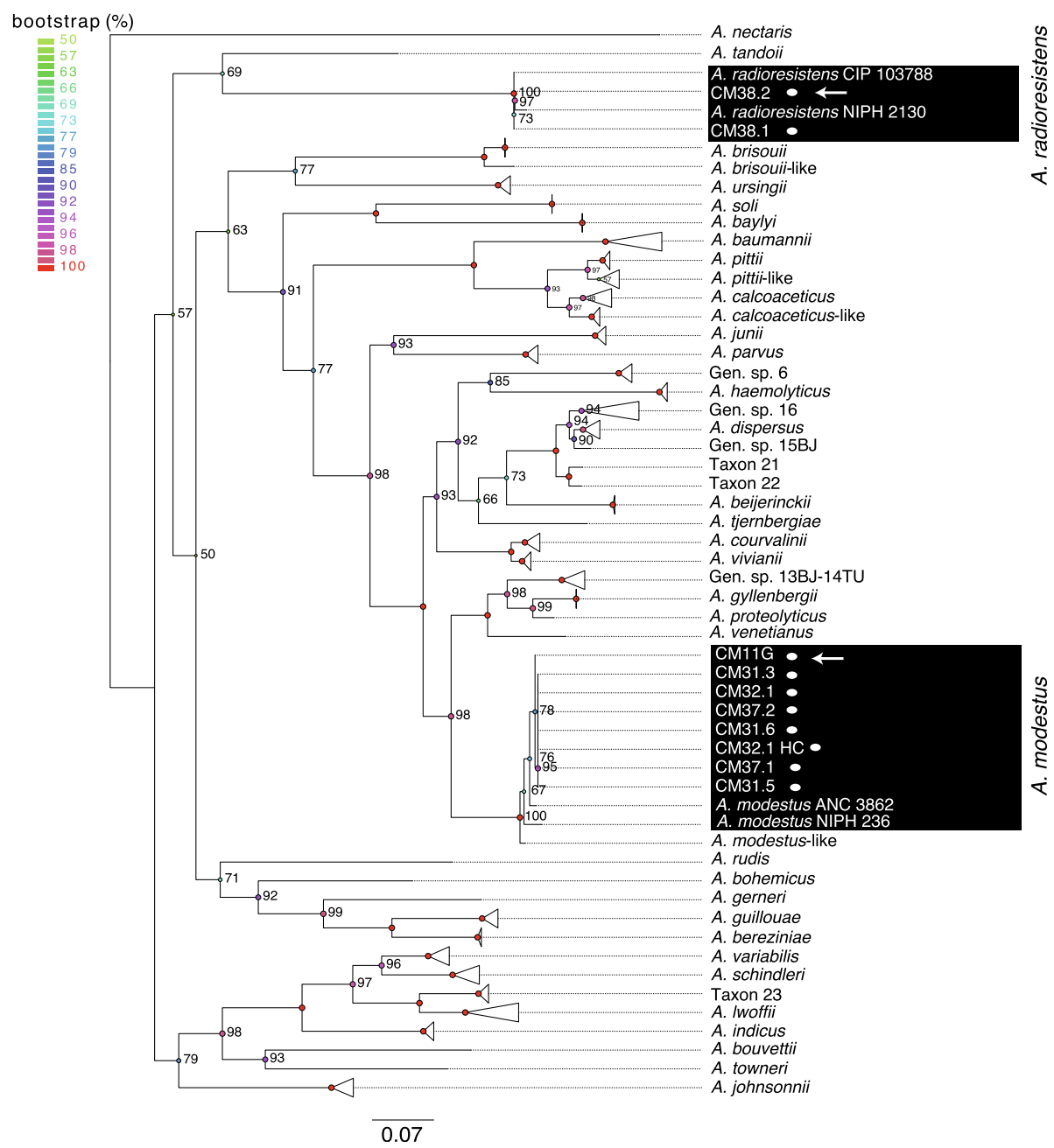

Fig. 1 Phylogenetic tree of Acinetobacter strains based on recA gene sequences. Triangles mark groups of taxa that are from the same species. The scale bar represents the average number of substitutions per site 


\section{Whole-genome sequencing}

One strain of each cluster was selected for sequencing: CM11G for A. modestus and CM38.2 for A. radioresistens - with the Illumina HiSeq 2000 technology (paired-end libraries) [11]. We also sequenced $A$. radioresistens CM38.2 strain (accession number SRR5351953) using the PacBio technology. Annotation with RAST gives 2968 coding DNA sequences (CDS) and 3104 CDS for A radioresistens CM38.2 paired-end and PacBio respectively. This latter annotation was used for the genomic comparative analysis. The distribution of the genes of CM11G and CM38.2 is listed in the Additional file 4: Table S2 according to the functional categories given by RAST.

\section{Acinetobacter Core-genomes}

In order to get an idea of the core-genome based on the sequences of $A$. modestus $\mathrm{CM} 11 \mathrm{G}$ and $A$. radioresistens CM38.2, a core-genome of 133 Acinetobacter strains [18] plus our two strains CM11G and CM38.2 was built (Table 2). The core-genome of the 135 genomes of Acinetobacter consists of 945 families of homologous proteins. A phylogenetic tree based on these proteins confirmed the identification of the two strains, since $A$. radioresistens CM38.2 is included is the $A$. radioresistens clade, and CM11G in the $A$. modestus clade (Fig. 2 and Additional file 5: Figure $\mathrm{S} 3$ ). The average nucleotide identity (ANIb) allows to putatively classify bacterial strains in the same species. It is currently admitted that an ANIb value of more than 95\% is strong indication that strains belong to the same species [31]. The values of ANIb between CM11G and two $A$. modestus strains (ANC 3862, NIPH 236) are higher than $96 \%$. The values of ANIb for the comparisons between CM38.2 and two A. radioresistens strains (NIPH 2130, CIP 103788) are higher than 98\%. These results are consistent with those obtained with the phylogenetic tree based on the Acinetobacter core-genome (Table 3), and suggest that the two strains are part of well-defined Acinetobacter species.

The core-genomes of species $A$. modestus and $A$. radioresistens were built using the three strains available for each (two published and one sequenced by us, see

Table $\mathbf{2}$ Core genome of Acinetobacter

\begin{tabular}{|c|c|c|c|c|}
\hline & $\begin{array}{l}\text { Number of } \\
\text { genomes }\end{array}$ & $\begin{array}{l}\text { Average } \\
\text { number of } \\
\text { gene families }\end{array}$ & $\begin{array}{l}\text { Smallest } \\
\text { proteome }\end{array}$ & $\begin{array}{l}\text { Number of core } \\
\text { gene families (\% of } \\
\text { the smallest) }\end{array}$ \\
\hline Acinetobacter & $133^{a}$ & 3523 & 2562 & 950 \\
\hline Acinetobacter & $135^{\mathrm{b}}$ & 3529 & 2562 & 945 \\
\hline $\begin{array}{l}\text { Acinetobacter } \\
\text { radioresistens }\end{array}$ & $3^{c}$ & 2991 & 2936 & 2391 (81\%) \\
\hline $\begin{array}{l}\text { Acinetobacter } \\
\text { modestus }\end{array}$ & $3^{d}$ & 3930 & 3347 & 2637 (79\%) \\
\hline
\end{tabular}

Methods). They contain 2637 and 2391 of orthologous proteins families, corresponding to $79 \%$ and $81 \%$ of the genomes of our strains for respectively $A$. modestus and A. radioresistens (Table 2 ).

\section{Acinetobacter pan-genomes}

The analysis of the core genome showed that both genomes contain many genes absent from the core genome of their species. In order to compare the variability between strains, we analyzed their pan-genomes (see Methods). At the genus level, the 135 Acinetobacter strains contained 30,080 protein families (identified using a threshold of $40 \%$ similarity, Table 4). At the species level, using a threshold of $80 \%$ similarity, we identified 3745 and 5264 proteins for $A$. radioresistens and $A$. modestus respectively (Fig. 3 and Table 4). The genomes of the strains from the crypts were, in both cases, larger than the ones of the other strains of the same species. Accordingly, they have more strainspecific genes than the others, which may provide traits involved in the adaptation to their specific niche.

\section{Antibiotic resistance profiles}

In order to get a better phenotypic characterization of the ten isolated strains, their antibiotic resistance was analyzed using a panel of 32 antimicrobials agents usually tested for non-fermentative Gram-negative bacteria. As for the biochemical identification and the phylogenetic analysis, the ten strains could be divided into two clusters. The eight $A$. modestus strains were resistant to streptomycin, spectinomycin, and latamoxef (cephalosporin family). The two $A$. radioresistens were sensitive to these antibiotics but resistant to chloramphenicol, unlike the $A$. modestus strains (Additional file 6: Table S3).

Aminoglycoside-modifying enzymes mediate the resistance to aminoglycosides such as streptomycin. A comparative analysis of antibiotic resistance genes performed on the two selected sequenced strains indicated the presence of an $\mathrm{O}$-adenylyltransferase $(\operatorname{aad} A)$ in the genome of $A$. modestus whereas the gene encoding this enzyme was absent in the genome of $A$. radioresistens CM38.2. This could explain the resistance to streptomycin and spectinomycin of the former strain. RarD, encoding a chloramphenicol-sensitive protein, and cat genes encoding a chloramphenicol acetyltransferase were present in the two genomic sequences even if $A$. modestus CM11G is sensitive to this antibiotic. Other resistance mechanisms, such as efflux pumps, may contribute to chloramphenicol resistance in $A$. radioresistens, such as the gene $m d f A$ coding for a multidrug/ chloramphenicol efflux transport belonging to the MFS (major facilitator superfamily) which is present in both strains. The RND (resistance-nodulation-division) family of efflux pumps is often described in genomes of 


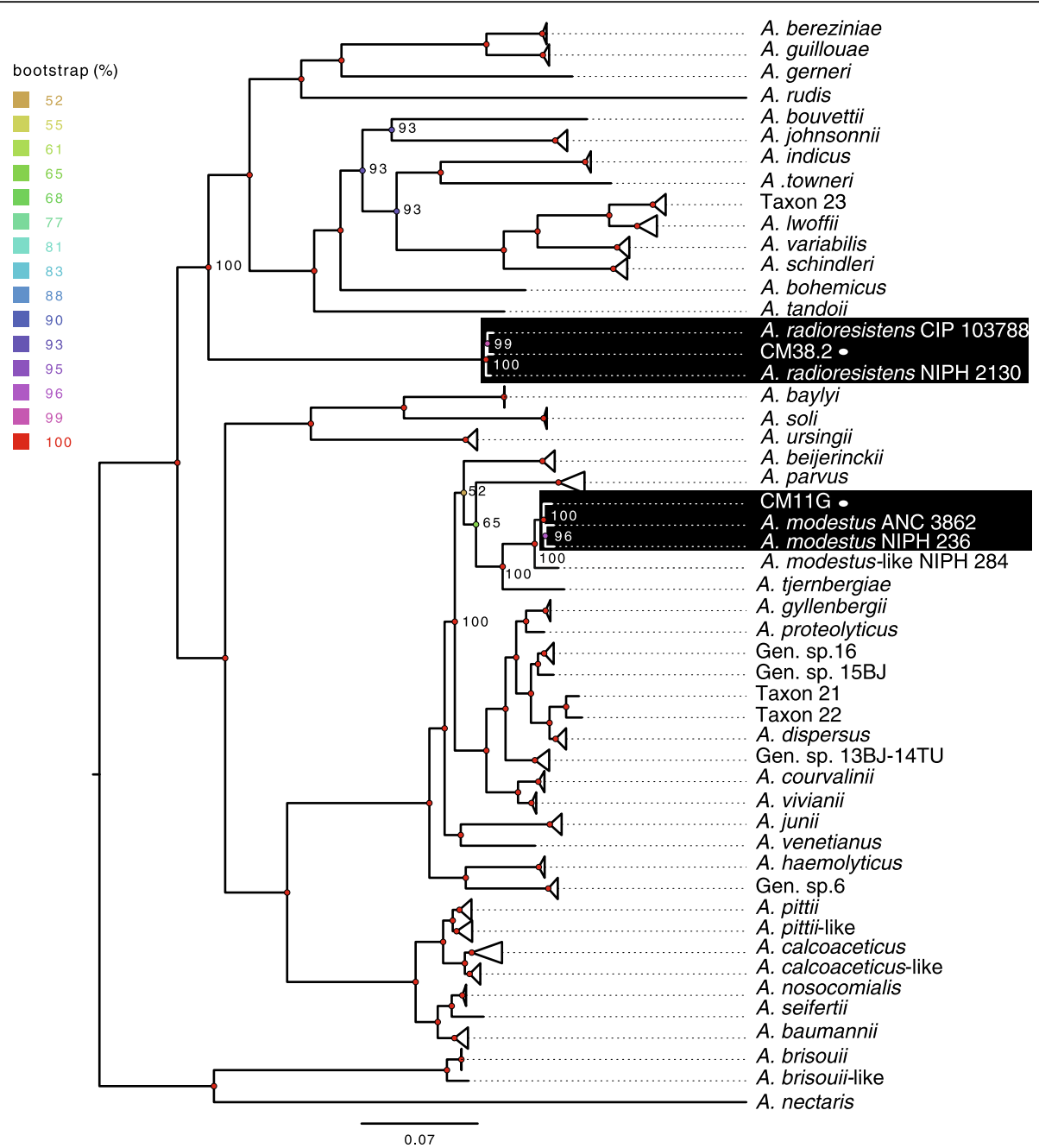

Fig. 2 Phylogeny of the Acinetobacter genus based on the alignment of the protein families of the core-genome. Triangles mark groups of taxa that are from the same species. The scale bar represents the average number of substitutions per site

Table 3 Average Nucleotide Identity (ANIb) in percent between the $A$. radioresistens strains $(A)$ and between $A$. modestus strains (B)

A. A. radioresistens Average nucleotide identity

$\begin{array}{llll} & \text { CM38.2 } & \text { NIPH2130 } & \text { CIP103788 } \\ \text { CM38.2 } & - & 98.06 & 98.11 \\ \text { NIPH2130 } & 98.26 & - & 98.30 \\ \text { CIP103788 } & 98.12 & 98.06 & -\end{array}$

B. A. modestus Average nucleotide identity

\begin{tabular}{llll} 
& ANC3862 & NIPH236 & CM11G \\
ANC3862 & - & 96.80 & 96.72 \\
NIPH236 & 96.81 & - & 96.66 \\
CM11G & 96.80 & 96.75 & - \\
\hline
\end{tabular}

The ANIb based on BLAST+ were performed with the software JSpecies pathogenic MDR strains of $A$. baumannii, mainly the AdeABC system [32]. However these genes were not present in the two sequenced strains analyzed in this study. But other RND type efflux pumps and many genes involved in the resistance to heavy metals such as arsenate, cobalt, zinc, cadmium and also to copper were found in the two genomes (Additional file 7: Table S4).

\section{Xenobiotic metabolism}

The intestinal microbiota is able to metabolize xenobiotics, including drugs, and thereby modulate their toxicological and pharmacological properties [33]. We wondered if some operons or genes involved in this process were present in our selected strains through the annotation tables. Both $A$. modestus CM11G and A. radioresistens CM38.2 carry the operon ben $A B C D E$ encoding benzoate 1,2-dioxygenase alpha $(\operatorname{ben} A)$ and beta $(\operatorname{ben} B)$ subunits with sequence homologies between themselves of 89.9 and 85.8 respectively 
Table 4 Pan genome of Acinetobacter

\begin{tabular}{llllll}
\hline & $\begin{array}{l}\text { Number of } \\
\text { genomes }\end{array}$ & $40 \%$ & $50 \%$ & $70 \%$ & $80 \%$ \\
\hline Acinetobacter & $135^{\mathrm{a}}$ & 30,080 & 35,274 & 53,163 & 71,471 \\
Acinetobacter radioresistens & $3^{\mathrm{b}}$ & 3489 & 3577 & 3695 & 3745 \\
Acinetobacter modestus & $3^{\mathrm{c}}$ & 4908 & 5049 & 5187 & 5264 \\
\hline
\end{tabular}

${ }^{a} 133$ strains and $\mathrm{CM} 11 \mathrm{G}$ and $\mathrm{CM} 38.2$;

${ }^{\mathrm{b}} \mathrm{CM} 38.2+\mathrm{NIPH} 2130+\mathrm{CIP} 103788 ;{ }^{\mathrm{C}} \mathrm{CM} 11 \mathrm{G}+\mathrm{ANC} 3862+\mathrm{NIPH} 236$

(Fig. 4A). The operon ant $A B C$ encoding the anthranilate dioxygenase, which catalyzes catechol formation, is present in the genomic sequence of $A$. radioresistens CM38.2, in the available genomic sequence of $A$. radioresistens $\mathrm{SH} 164$ (accession number NZ_GG705131), and in the two $A$. radioresistens strains used for the core-genome analysis. In contrast, it is absent in A. modestus CM11G. It should be noted that the ant $A B C$ operon is also present in the genome of various Acinetobacter species such as A. baumannii (AB307 and ATCC 17978), A. pittii PHEA-2 (accession number CP002177) and A. baylyi ADP1 [34]. Moreover, the phenol hydroxylase gene cluster $d m p K L M N O P$, which convert phenol to catechol, is absent in the genome of $A$. modestus CM11G whereas it is present in $A$. radioresistens CM38.2, A. radioresistens SH164 and A. pittii PHEA-2 (Fig. 4B) [4]. The organization of phenol degradation genes in these strains indicates a high degree of similarity, with a homology of $100 \%$ with A. radioresistens SH164 and around $80 \%$ with $A$. calcoaceticus. These findings indicate that the strains isolated from murine intestinal crypts are able to metabolize xenobiotics, even if at different levels.

\section{Secretion systems}

A diversity of secretion systems were described in Gram-negative bacteria and in some strains of Acinetobacter such a Type I, Type II and Type VI secretion systems that are known to be involved in pathogenicity or in supporting survival in a complex microbial community $[35,36]$. The T1SS secreted agglutinin RTX was found in the genomic sequence of $A$. radioresistens CM38.2 and shared a sequence identity of $40 \%$ with the biofilm-associated protein (Bap) of A. baumannii AB0057. Bap plays a role in the adhesion to host cell and in the maintenance of the biofilm. The gene encoding TolC, a porin at the outer membrane that is part of T1SS and drug-efflux pumps [37], also involved in biofilm formation, is present in the genomic sequences of $A$. modestus CM11G and A. radioresistens CM38.2. Quantitative analysis of biofilm formed by these two strains indicates that both A. modestus CM11G and A. radioresistens CM38.2 are capable to induce biofilm after $24 \mathrm{H}$ of incubation even if $A$. modestus CM11G is a stronger inducer of biofilm than A. radioresistens CM38.2. After $48 \mathrm{H}$ of culture the ability of $A$. radioresistens CM38.2 is weaker, whereas $A$. modestus CM11G remains as strong as A. baumannii CIP $70.34^{\mathrm{T}}$ (Additional file 8: Figure S4).

Genes encoding general secretion pathway proteins (gsp) that are associated with the type II secretion system (T2SS) [38] were found in the two strains. The operon encoding the twin-arginine translocation proteins (TatABC), involved in the export of folded proteins across the cytoplasmic membrane of bacteria, also known as sec-independent translocase proteins, was found in the genomic sequences of $A$. modestus CM11G and A. radioresistens CM38.2.

A type VI secretion system (T6SS) is present in many Acinetobacter isolates, environmental or clinical strains [39] and could be involved in host colonization [40]. This secretion system was not found in the genomic sequences of A. modestus CM11G and A. radioresistens CM38.2. Interestingly, a T6SS is present in the two A. radioresistens strains NIPH 2130 and CIP 103788 suggesting an adaptation of the strain CM38.2 to its niche.

xSeveral genes involved in adhesion and biofilm formation such as type IV pili [41] are represented in both selected strains $A$. modestus $\mathrm{CM} 11 \mathrm{G}$ and $A$. radioresistens CM38.2. Even if Acinetobacter name means non-motile rod, some species are able of bacterial locomotion through

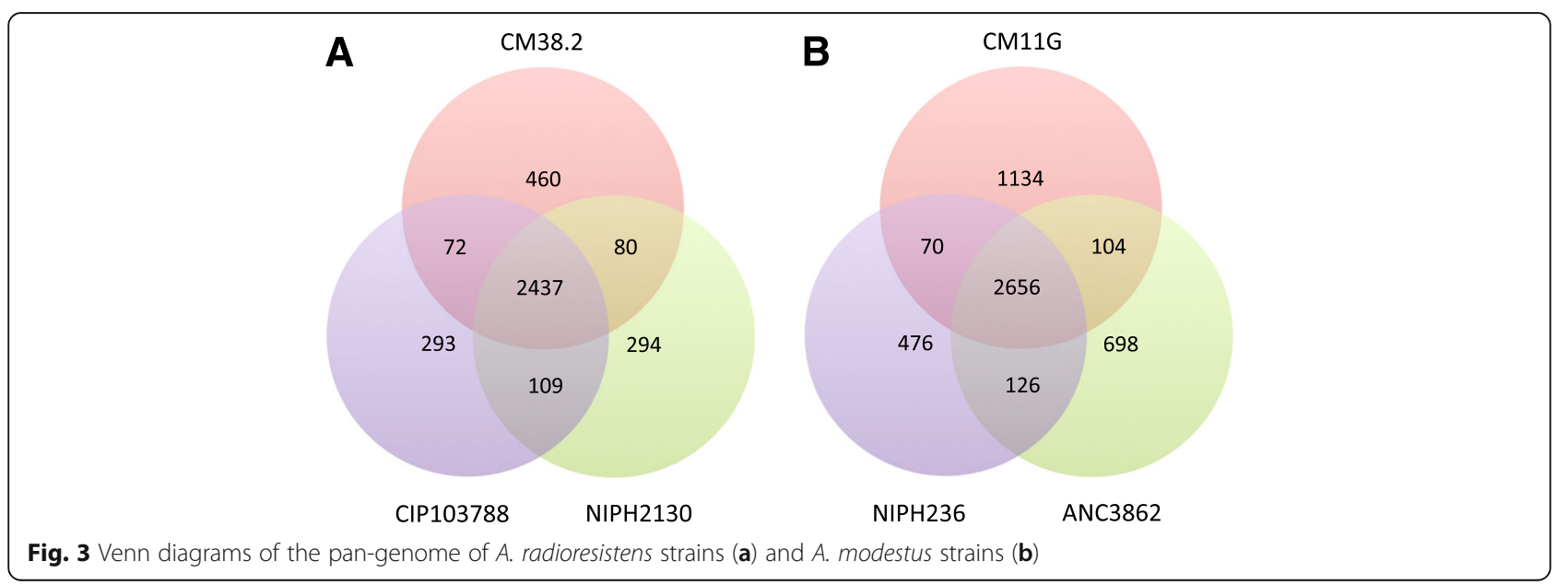




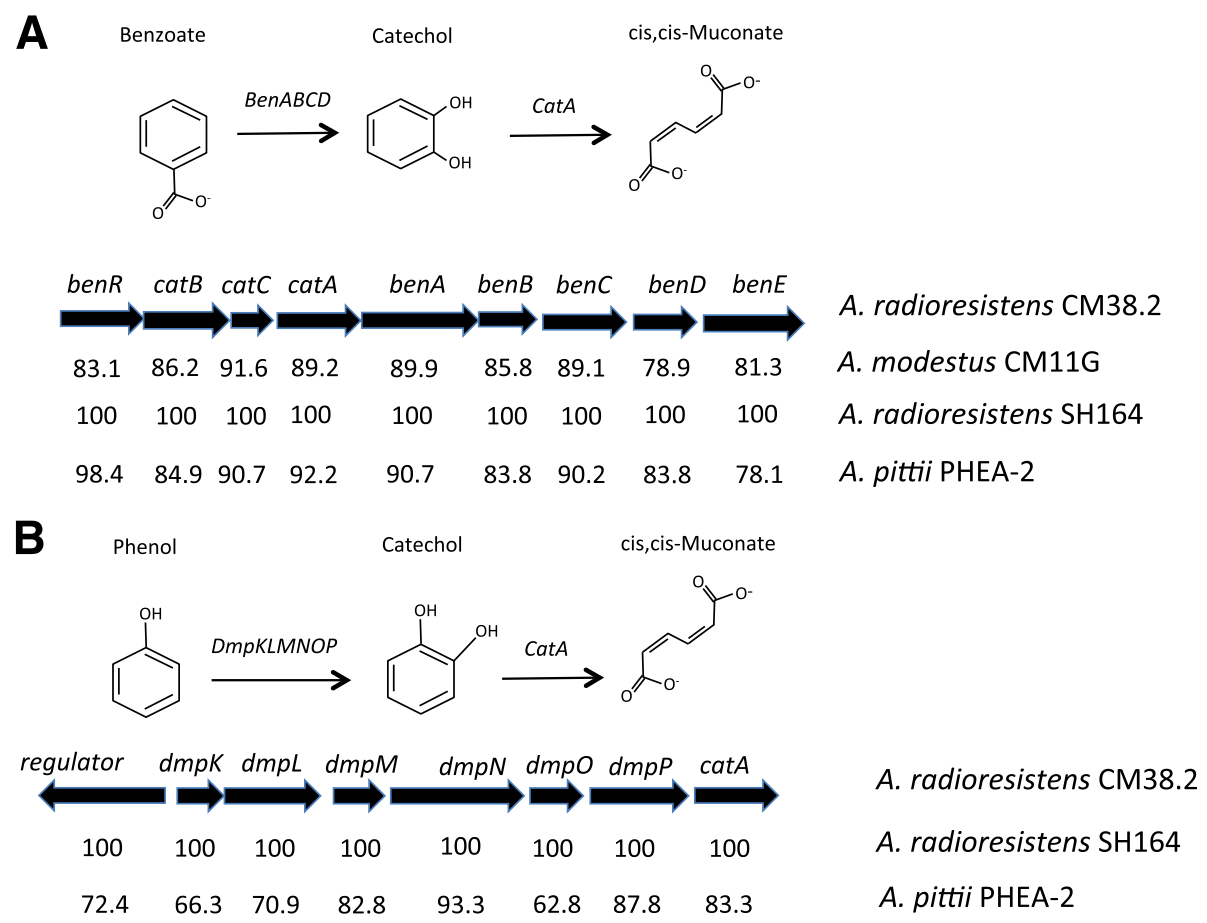

Fig. 4 Genetic organization and conservation of the BenABCDE cluster (a), and of the phenol degradation operon (b). The percentage of nucleotides identity between the strains for each gene is indicated

Type IV dependent twitching motility [42]. Many Acinetobacter species harbor the genes encoding the proteins necessary for a functional Type IV pili system [43, 44]. Similarly 22 genes among the 24 listed are both present in the genomic sequences of $A$. modestus CM11G and $A$. radioresistens CM38.2 (Table 5). The Type IV pili system is also involved in natural transformation in Acinetobacter, where transformation is tightly associated with mobility [45]. Overall, these results indicate that the two selected strains from murine intestinal crypts shared many secretion systems that could allow the colonization of this particular niche.

\section{Siderophores}

Iron is an essential element for the growth of a large number of bacteria [46]. Several mechanisms to acquire iron have been developed by bacteria, including siderophores. Moreover these siderophores are considered as virulence factors for pathogenic bacteria such as A. baumannii that encodes for the highly conserved acinetobactin $[47,48]$. A siderophore cluster was found in the genome of $A$. radioresistens CM38.2 whereas some genes were missing in the genome of $A$. modestus CM11G (Fig. 5). Another cluster of four genes is also present in A. radioresistens CM38.2 but absent in the $A$. modestus CM11G strain (Additional file 7: Table S4). This cluster encodes proteins involved in the siderophore $\mathrm{S}$ biosynthesis. The same clusters for iron uptake are also present in the genome sequence of
A. radioresistens $\mathrm{SH} 164$ with a sequence similarity of $100 \%$ except for one of the genes (Fig. 5). This reflects an iron metabolic variability for the two Acinetobacter strains.

\section{Discussion}

In this study, we characterized the genomic features of two Acinetobacter isolated from murine colonic crypts and belonging to the Crypt Specific Core Microbiota (CSCM). They were isolated from each of the two large clusters of Acinetobacter strains that can be distinguished by their biochemical profiles: $A$. modestus and $A$. radioresistens. These two clusters could also be discriminated based on their antibiotic resistance. In contrast to many pathogenic Acinetobacter bacteria such as $A$. baumannii that possess a T6SS [40], the T6SS was not encoded by the genome of $A$. modestus and $A$. radioresistens. However, genes encoding proteins involved in Type I secretion system, Type II secretion system and Type IV pili system are present in the genomic sequences of the selected strains. Many others genes involved in heavy metal resistance as well as multidrug resistance efflux pumps are encoded by these strains.

The two Acinetobacter shared also some operons such as the ben $A B C D E$ involved in benzoate degradation whereas the operon involved in phenol degradation is absent in the A. modestus strain. Does CSCM "gate keeper" exert protection of the epithelial regenerative 
Table 5 Genes involved in Type IV pilus apparatus in the genome of A. modestus CM11G and A. radioresistens CM38.2

\begin{tabular}{|c|c|c|c|}
\hline Function & A. modestus CM11G & A. radioresistens $\mathrm{CM} 38.2$ & Sequence Identity (\%) \\
\hline 3-dehydroquinate synthase (EC 4.2.3.4) & 1 & 1 & 82.40 \\
\hline Fimbrial assembly protein FimB & 1 & 0 & \\
\hline Leader peptidase (Prepilin peptidase) & 1 & 1 & 81.12 \\
\hline Multimodular transpeptidase-transglycosylase & 1 & 1 & 79.35 \\
\hline $\mathrm{N}$-methyltransferase & 1 & 1 & 81.12 \\
\hline Twitching motility protein PilG & 1 & 1 & 99.21 \\
\hline Twitching motility protein PilH & 1 & 1 & 85.83 \\
\hline Twitching motility protein PilT & 1 & 1 & 57.81 \\
\hline Type IV fimbriae expression regulatory protein PilR & 0 & 1 & \\
\hline Type IV fimbrial assembly protein PilC & 1 & 1 & 91.67 \\
\hline Type IV fimbrial assembly, ATPase PilB & 1 & 1 & 80.42 \\
\hline Type IV fimbrial biogenesis protein FimT & 1 & 1 & 39.88 \\
\hline Type IV fimbrial biogenesis protein PilV & 1 & 1 & 38.83 \\
\hline Type IV fimbrial biogenesis protein PilW & 1 & 1 & 36.89 \\
\hline Type IV fimbrial biogenesis protein PilX & 1 & 1 & 30.53 \\
\hline Type IV fimbrial biogenesis protein PilY1 & 1 & 1 & 30.15 \\
\hline Type IV pili signal transduction protein Pill & 1 & 1 & 78.65 \\
\hline Type IV pilin PilA & 1 & 1 & 80.49 \\
\hline Type IV pilus biogenesis protein PilJ & 1 & 1 & 77.99 \\
\hline Type IV pilus biogenesis protein PilM & 1 & 1 & 85.81 \\
\hline Type IV pilus biogenesis protein PilN & 1 & 1 & 78.24 \\
\hline Type IV pilus biogenesis protein PilO & 1 & 1 & 70.45 \\
\hline Type IV pilus biogenesis protein PilP & 1 & 1 & 74.57 \\
\hline Type IV pilus biogenesis protein PilQ & 1 & 1 & 72.78 \\
\hline
\end{tabular}

1: presence of the feature; 0 : absence of the feature. The percentage number represents the identity in the sequences of the two genomes
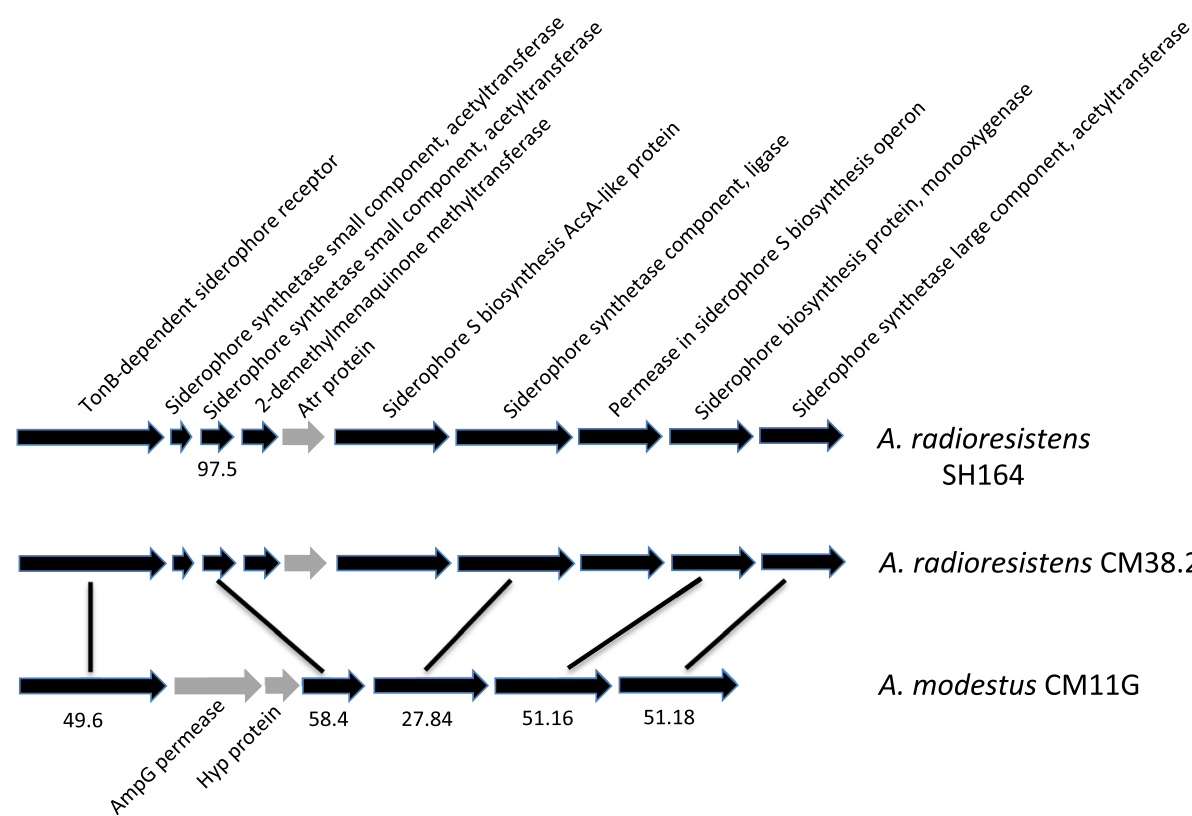

A. radioresistens $\mathrm{CM} 38.2$

A. modestus $\mathrm{CM} 11 \mathrm{G}$

Fig. 5 Genetic organization of the siderophore cluster found in A. radioresistens CM38.2. The percentage of nucleotide identity with A. radioresistens SH164 and A. modestus CM11G are indicated 
apparatus against the (geno)toxic potential of metabolic byproducts of the microbiota and xenobiotics? Undigested dietary fibers and endogenous residues are metabolized by the gut microbiota and some of the by-products of this microbial metabolism are involved in tumor promotion (secondary bile acids, anaerobic tryptophan degradation products: indoles, ammonia), mutagenesis (fecapenaenes) or oncogenesis ( $\mathrm{N}$-nitrosocompounds). Numerous bacterial enzymes responsible for the production of carcinogens have been identified. The protective effect of certain bacterial species is also recognized, encompassing carcinogen binding, detoxification of methylmercury, formation of isoflavones [49]. Regarding xenobiotics, there is a broad range of bacterial dehalogenases that catalyze the cleavage of carbon-halogen bonds, which is a key step in aerobic mineralization pathways of many potentially carcinogenic halogenated compounds that occur as environmental pollutants [50].

This study sheds new light on genomic features involved in xenobiotic metabolism that could play a crucial role in the protection of colonic crypts that harbor the intestinal stem cells.

\section{Conclusions}

In this study, we used whole-genome sequencing to characterize Acinetobacter isolated from murine colonic crypts. We performed genomic analysis of two isolates belonging to two different species in comparison to available Acinetobacter genomes in public databases. Our results shed new light on genomic features involved in xenobiotic metabolism that could play a crucial role in the protection of colonic crypts that harbor the intestinal stem cells.

\section{Additional files}

Additional file 1: Table S1. Sequence of primers used for identification of the strains based on the sequences of 165 rRNA and recA. (DOCX $33 \mathrm{~kb}$ )

Additional file 2: Figure S1. Whole genome comparative alignment of A. radioresistens CM38.2. The genome sequence is presented horizontally with the scale showing the sequence coordinates and the conserved shared synteny represented as the colored blocks which are connected across genomes. Upper panel: PacBio sequencing; lower panel: Illumina paired-end sequencing. (PDF $80 \mathrm{~kb}$ )

Additional file 3: Figure S2. Complete phylogenetic tree of Acinetobacter strains based on 16S rRNA gene sequences. The scale bar represents the average number of substitutions per site. (PDF $586 \mathrm{~kb}$ )

Additional file 4: Table S2. Distribution of the genes of $A$. modestus CM11G and A. radioresistens CM38.2 following the functional categories obtained following RAST annotations. (DOCX $73 \mathrm{~kb}$ )

Additional file 5: Figure S3. Complete phylogenetic tree of the Acinetobacter genus based on the alignment of the protein families of the core-genome. The scale bar represents the average number of substitutions per site. (PDF $302 \mathrm{~kb}$ )

Additional file 6: Table S3. Antibiotic resistance pattern of the 10 Acinetobacter isolates. S: sensitive; I: intermediate; R: resistant. This panel of 32 antimicrobials agents is usually tested for non-fermentative Gramnegative bacteria. (DOCX $106 \mathrm{~kb}$ )

Additional file $\mathbf{7}$ Table S4. Comparison of the genomes of $A$. modestus CM11G and A. radioresistens CM38.2 based on their functional categories according to RAST classification into subsystems. 1: presence of the feature; 0: absence of the feature. (XLSX $97 \mathrm{~kb}$ )

Additional file 8: Figure S4. Quantification of biofilm formation. Bacteria were incubated at $37^{\circ} \mathrm{C}$ in Trypticase-Soy broth in polystyrene plate for $24 \mathrm{H}(\mathrm{A})$ or $48 \mathrm{H}(\mathrm{B})$. Data are expressed as mean \pm Standard deviation, $n=6$ in each group. ${ }^{* *}, P<0.001$ versus biofilm formation by Escherichia coli DH5a. Control: uninoculated wells. (PDF 56 kb)

\section{Abbreviations}

CSCM: Crypt Specific Core Microbiota; MDR: multi-drug resistance; recA: recombinase A; RND: resistance-nodulation-division; T6SS: Type VI secretion system

\section{Acknowledgements}

We wish to thanks François-Xavier Weill and the team of the Enteric Pathogenic Bacteria Research and Expertise Unit at the Institut Pasteur for the experiment of antibiotic resistance. We wish also to thank the Biological Resource Center of Institut Pasteur for the gift of Acinetobacter baumannii CIP $70.34^{\top}$.

\section{Availability of data and materials}

Sequence data that support the findings of this study have been deposited in GenBank under the accession numbers: LACJ00000000, LATS00000000 and SRR5351953.

\section{Funding}

This work was supported by the European Research Council (PJS Advanced Grant 339,579). The European Research Council did not play any role in the design of this study or in the collection, analysis or interpretation of the data or in writing the manuscript.

\section{Authors' contributions}

PJS and TP conceived the study; AS and MT annotated genomes, and performed analysis; AS, MT, EPCR and TP wrote the manuscript; EPCR, RT VP and SB helped with the genome analysis; CM and TP identified and characterized the strains. All authors read and approved the final manuscript.

\section{Ethics approval and consent to participate}

Six- to ten- week old C57BL/6 mice from Elevage Janvier (Le Genest Saint Isle, France) were used in this study. Dixit. Animals were housed in the Institut Pasteur animal facility accredited by the French Ministry of Agriculture for performing experiments on live rodents. Work on animals was performed in compliance with French and European regulations on care and protection of laboratory animals (EC Directive 2010/63, French Law 2013-118, February 6th, 2013). All experiments were approved by the Ethics Committee \#89 and registered under the reference 2013-0030.

\section{Consent for publication}

Not applicable.

\section{Competing interests}

The authors declare that they have no competing interests.

\section{Publisher's Note}

Springer Nature remains neutral with regard to jurisdictional claims in published maps and institutional affiliations.

\section{Author details}

${ }^{1} U$ unité de Pathogénie Microbienne Moléculaire, INSERM U1202, Institut Pasteur, Paris, France. ${ }^{2}$ Unité de Génomique Evolutive des Microbes, CNRS, UMR3525, Institut Pasteur, Paris, France. ${ }^{3}$ Unité de Pathogénie Microbienne Moléculaire, INSERM U1202, Imagopole Citech, Institut Pasteur, Paris, France. ${ }^{4}$ Chaire de Microbiologie et Maladies Infectieuses, Collège de France, Paris, France. 
Received: 3 April 2017 Accepted: 6 July 2017

\section{Published online: 11 July 2017}

\section{References}

1. Nemec A. Classification and nomenclature of the genus Acinetobacter. http://apps.szu.cz/anemec/Classification.pdf. Updated: December 4, 2016.

2. Seifert H, Dijkshoorn L. Overview of the Microbial Characteristics, Taxonomy, and Epidemiology. In: Bendinelli M, Friedman H, editors. Acinetobacter Biology and Pathogenesis. N Y: Springer; 2009. p. 14-45.

3. Jung J, Madsen EL, Jeon CO, Park W. Comparative genomic analysis of Acinetobacter oleivorans DR1 to determine strain-specific genomic regions and gentisate biodegradation. Appl Environ Microbiol. 2011;77(20):7418-24. doi:10.1128/AEM.05231-11.

4. Zhan Y, Yan Y, Zhang W, Chen M, Lu W, Ping S, Lin M. Comparative analysis of the complete genome of an Acinetobacter calcoaceticus strain adapted to a phenol-polluted environment. Res Microbiol. 2012;163(1):36-43. doi:10. 1016/j.resmic.2011.10.006

5. Pédron T, Mulet C, Dauga C, Frangeul L, Chervaux C, Grompone G, Sansonetti PJ. A crypt-specific core microbiota resides in the mouse colon. MBio. 2012;3(3):e00116-2. doi:10.1128/mBio.00116-12.

6. Marteyn B, West NP, Browning DF, Cole JA, Shaw JG, Palm F, Mounier J, Prévost MC, Sansonetti $P$, Tang CM. Modulation of Shigella virulence in response to available oxygen in vivo. Nature. 2010;465(7296):355-8. doi:10. 1038/nature08970.

7. Baumann P. Isolation of Acinetobacter from soil and water. JBacteriol. 1968:96:39-42

8. Soussy CJ, Carret G, Cavallo JD, Chardon H, Chidiac C, Choutet P, Courvalin P, Dabernat H, Drugeon H, Dubreuil L, Goldstein F, Jarlier V, Leclercq R, Nicolas-Chanoine MH, Philippon A, Quentin C, Rouveix B, Sirot J. Report of the Antibiogram Committee of the French Microbiology Society, 2000-2001. Pathol Biol. 2000;48:832-71.

9. O'Toole GA, Pratt LA, Watnick PI, Newman DK, Weaver VB, Kolter R. Genetic approaches to study of biofilms. Methods Enzymol. 1999;310:91-109.

10. Tomaras AP, Dorsey CW, Edelmann RE, Actis LA. Attachment to and biofilm formation on abiotic surfaces by Acinetobacter Baumannii: involvement of a novel chaperone-usher pili assembly system. Microbiology. 2003;149(Pt 12):3473-84.

11. Saffarian A, Mulet C, Naito T, Bouchier C, Tichit M, Ma L, Grompone G, Sansonetti PJ, Pédron T. Draft Genome Sequences of Acinetobacter parvus CM11, Acinetobacter radioresistens CM38, and Stenotrophomonas maltophilia BR12, Isolated from Murine Proximal Colonic Tissue. Genome Announc. 2015;3(5):e01089-15. doi:10.1128/genomeA.01089-15.

12. Eid J, Fehr A, Gray J, Luong K, Lyle J, Otto G, Peluso P, Rank D, Baybayan P, Bettman B, Bibillo A, Bjornson K, Chaudhuri B, Christians F, Cicero R, Clark S, Dalal R, Dewinter A, Dixon J, Foquet M, Gaertner A, Hardenbol P, Heiner C, Hester K, Holden D, Kearns G, Kong X, Kuse R, Lacroix Y, Lin S, Lundquist P, Ma C, Marks P, Maxham M, Murphy D, Park I, Pham T, Phillips M, Roy J, Sebra R, Shen G, Sorenson J, Tomaney A, Travers K, Trulson M, Vieceli J, Wegener J, Wu D, Yang A, Zaccarin D, Zhao P, Zhong F, Korlach J, Turner S. Real-time DNA sequencing from single polymerase molecules. Science. 2009;323:133-8. doi:10.1126/science.1162986.

13. Chin CS, Alexander DH, Marks P, Klammer AA, Drake J, Heiner C, Clum A, Copeland A, Huddleston J, Eichler EE, Turner SW, Korlach J. Nonhybrid, finished microbial genome assemblies from long-read SMRT sequencing data. Nat Methods. 2013:10:563-9. doi:10.1038/nmeth.2474.

14. Darling ACE, Mau B, Blattner FR, Perna NT. Mauve: multiple alignment of conserved genomic sequence with rearrangements. Genome Res. 2004;14 1394-403.

15. Aziz RK, Bartels D, Best AA, DeJongh M, Disz T, Edwards RA, Formsma K, Gerdes S, Glass EM, Kubal M, Meyer F, Olsen GJ, Olson R, Osterman AL, Overbeek RA, McNeil LK, Paarmann D, Paczian T, Parrello B, Pusch GD, Reich C, Stevens R, Vassieva O, Vonstein V, Wilke A, Zagnitko O. The RAST server: rapid annotations using subsystems technology. BMC Genomics. 2008;9:75. doi:10.1186/1471-2164-9-75.

16. Overbeek R, Olson R, Pusch GD, Olsen GJ, Davis JJ, Disz T, Edwards RA, Gerdes S, Parrello B, Shukla M, Vonstein V, Wattam AR, Xia F, Stevens R. The SEED and the rapid annotation of microbial genomes using subsystems technology (RAST). Nucleic Acids Res. 2014;42(1):D206-14.

17. Vallenet D, Belda E, Calteau A, Cruveiller S, Engelen S, Lajus A, Le Fèvre F, Longin C, Mornico D, Roche D, Rouy Z, Salvignol G, Scarpelli C, Thil Smith AA, Weiman M, Médigue C. MicroScope_-an integrated microbial resource for the curation and comparative analysis of genomic and metabolic data. Nucleic Acids Res. 2013;41:D636-47.

18. Touchon M, Cury J, Yoon EJ, Krizova L, Cerqueira GC, Murphy C, Feldgarden M, Wortman J, Clermont D, Lambert T, Grillot-Courvalin C, Nemec A, Courvalin P, Rocha EP. 2014. The genomic diversification of the whole Acinetobacter genus: origins, mechanisms, and consequences. Genome Biol Evol. 2014;6(10):2866-82.

19. Touchon M, Hoede C, Tenaillon O, Barbe V, Baeriswyl S, Bidet P, Bingen E, Bonacorsi S, Bouchier C, Bouvet O, Calteau A, Chiapello H, Clermont O, Cruveiller S, Danchin A, Diard M, Dossat C, Karoui ME, Frapy E, Garry L, Ghigo JM, Gilles AM, Johnson J, Le Bouguénec C, Lescat M, Mangenot S, Martinez-Jéhanne V, Matic I, Nassif X, Oztas S, Petit MA, Pichon C, Rouy Z, Ruf CS, Schneider D, Tourret J, Vacherie B, Vallenet D, Médigue C, Rocha EP, Denamur E. Organised genome dynamics in the Escherichia coli species results in highly diverse adaptive paths. PLoS Genet. 2009;5(1):e1000344. doi: 10.1371/journal.pgen.1000344.

20. Dandekar T, Snel B, Huynen M, Bork P. Conservation of gene order: a fingerprint of proteins that physically interact. Trends Biochem Sci. 1998; 23(9):324-8.

21. Rocha EP, Touchon M, Feil EJ. Similar compositional biases are caused by very different mutational effects. Genome Res. 2006;16(12):1537-47.

22. Altschul SF, Madden TL, Schäffer AA, Zhang J, Zhang Z, Miller W, Lipman DJ. Gapped BLAST and PSI-BLAST: a new generation of protein database search programs. Nucleic Acids Res. 1997;25:3389-402.

23. Miele $V$, Penel $S$, Duret $L$. Ultra-fast sequence clustering from similarity networks with SiLiX. BMC Bioinformatics. 2011;12:116. doi:10.1186/14712105-12-116.

24. Nawrocki EP, Eddy SR. Infernal 1.1: 100-fold faster RNA homology searches. Bioinformatics. 2013;29(22):2933-5. doi:10.1093/bioinformatics/btt509.

25. Nawrocki EP, Burge SW, Bateman A, Daub J, Eberhardt RY, Eddy SR, Floden EW, Gardner PP, Jones TA, Tate J, Finn RD. Rfam 12.0: updates to the RNA families database. Nucleic Acids Res. 2015;43(Database issue):D130-7. doi:10. 1093/nar/gku1063.

26. Nguyen LT, Schmidt HA, von Haeseler A, Minh BQ. IQ-TREE: a fast and effective stochastic algorithm for estimating maximum-likelihood phylogenies. Mol Biol Evol. 2015;32(1):268-74. doi:10.1093/molbev/msu300.

27. Katoh K, Standley DM. MAFFT multiple sequence alignment software version 7: improvements in performance and usability. Mol Biol Evol. 2013; 30(4):772-80. doi:10.1093/molbev/mst010.

28. Criscuolo A, Gribaldo S. BMGE (block mapping and gathering with entropy): a new software for selection of phylogenetic informative regions from multiple sequence alignments. BMC Evol Biol. 2010;10:210. doi:10.1186/ 1471-2148-10-210.

29. Nemec A, Radolfova-Krizova L, Maixnerova M, Vrestiakova E, Jezek P, Sedo O. Taxonomy of haemolytic and/or proteolytic strains of the genus Acinetobacter with the proposal of Acinetobacter courvalinii sp. nov. (genomic species 14 sensu Bouvet \& Jeanjean), Acinetobacter dispersus sp. nov. (genomic species 17), Acinetobacter modestus sp. nov., Acinetobacter proteolyticus sp. nov. and Acinetobacter vivianii sp. nov. Int J Syst Evol Microbiol. 2016;66(4):1673-85. doi:10.1099/ijsem.0.000932.

30. Krawczyk B, Lewandowski K, Kur J. Comparative studies of the Acinetobacter genus and the species identification method based on the recA sequences. Mol Cell Probes. 2002;16(1):1-11.

31. Richter M, Rosselló-Móra R. Shifting the genomic gold standard for the prokaryotic species definition. Proc Natl Acad Sci U S A. 2009;106(45): 19126-31.

32. Yoon EJ, Courvalin P, Grillot-Courvalin C. RND-type efflux pumps in multidrug-resistant clinical isolates of Acinetobacter Baumannii: major role for AdeABC overexpression and AdeRS mutations. Antimicrob Agents Chemother. 2013;57(7):2989-95. doi:10.1128/AAC.02556-12.

33. Claus SP, Guillou H, Ellero-Simatos S. The gut microbiota: a major player in the toxicity of environmental pollutants? Npj biofilms and microbiomes; 2016. doi:10.1038/npjbiofilms.2016.3.

34. Eby DM, Beharry ZM, Coulter ED, Kurtz DM, Neidl E. Characterization and evolution of anthranilate 1,2-dioxygenase from Acinetobacter sp. strain ADP1. J. Bacteriol. 2001;183:109-18. doi:10.1128/JB.183-1.109-118.2001.

35. Elhosseiny NM, El-Tayeb OM, Yassin AS, Lory S, Attia AS. The secretome of Acinetobacter Baumannii ATCC 17978 type II secretion system reveals a novel plasmid encoded phospholipase that could be implicated in lung colonization. Int J Med Microbiol. 2016;306(8):633-41. doi:10.1016/j.jijmm 2016.09.006. 
36. Weber BS, Hennon SW, Wright MS, Scott NE, de Berardinis V, Foster $\mathrm{L}$, Ayala JA, Adams MD, Feldman MF. Genetic dissection of the type VI secretion system in Acinetobacter and identification of a novel peptidoglycan hydrolase, TagX, required for its biogenesis. MBio. 2016;7(5): e01253-16. doi:10.1128/mBio.01253-16.

37. Koronakis V, Eswaran J, Hughes C. Structure and function of TolC: the bacterial exit duct for proteins and drugs. Annu Rev Biochem. 2004;73:467-89.

38. Douzi B, Filloux A, Voulhoux R. On the path to uncover the bacterial type II secretion system. Philos Trans R Soc Lond Ser B Biol Sci. 2012;367(1592): 1059-72. doi:10.1098/rstb.2011.0204.

39. Weber BS, Miyata ST, Iwashkiw JA, Mortensen BL, Skaar EP, Pukatzki S, Feldman MF. Genomic and functional analysis of the type VI secretion system in Acinetobacter. PLoS One. 2013;8(1):e55142. doi:10.1371/journal. pone.0055142.

40. Repizo GD, Gagné S, Foucault-Grunenwald ML, Borges V, Charpentier X, Limansky AS, Gomes JP, Viale AM, Salcedo SP. Differential role of the T6SS in Acinetobacter Baumannii virulence. PLoS One. 2015;10(9):e0138265. doi:10. 1371/journal.pone.0138265.

41. Vidal R, Dominguez M, Urrutia H, Bello H, Gonzalez G, Garcia A, Zemelman R. Biofilm formation by Acinetobacter Baumannii. Microbios. 1996;86(346):49-58.

42. Harding CM, Tracy EN, Carruthers MD, Rather PN, Actis LA, Munson RS Jr. Acinetobacter baumannii strain M2 produces type IV pili which play a role in natural transformation and twitching motility but not surface-associated motility. MBio. 2013;4(4):e00360-13. doi:10.1128/mBio.00360-13.

43. Antunes $L C$, Imperi F, Carattoli A, Visca P. Deciphering the multifactorial nature of Acinetobacter Baumannii pathogenicity. PLoS One. 2011;6(8):e22674.

44. Peleg AY, de Breij A, Adams MD, Cerqueira GM, Mocali S, Galardini M, Nibbering PH, Earl AM, Ward DV, Paterson DL, Seifert H, Dijkshoorn L. The success of acinetobacter species; genetic, metabolic and virulence attributes. PLoS One. 2012;7(10):e46984. doi:10.1371/journal.pone.0046984.

45. Wilharm G, Piesker J, Laue M, Skiebe E. DNA uptake by the nosocomial pathogen Acinetobacter Baumannii occurs during movement along wet surfaces. J Bacteriol. 2013;195(18):4146-53. doi:10.1128/JB.00754-13.

46. Symeonidis A, Marangos M. Iron and microbial growth. In : Insight and control of Infectious disease in global scenario. InTech. 2012:289-330. doi: 10.5772/34760.

47. Penwell WF, DeGrace N, Tentarelli S, Gauthier L, Gilbert CM, Arivett BA, Miller AA, Durand-Reville TF, Joubran C, Actis LA. Discovery and characterization of new Hydroxamate Siderophores, Baumannoferrin a and B, produced by Acinetobacter Baumannii. Chembiochem. 2015; doi:10.1002/ cbic.201500147.

48. Antunes LC, Imperi F, Towner KJ, Visca P. Genome-assisted identification of putative iron-utilization genes in Acinetobacter Baumannii and their distribution among a genotypically diverse collection of clinical isolates. Res Microbiol. 2011;162(3):279-84. doi:10.1016/j.resmic.2010.10.010.

49. Hughes R, Rowland IR. Metabolic activities of the gut microflora in relation to cancer. Microbial Ecol in Health and Dis. 2000;Suppl 2:179-185.

50. Janssen DB, Dinkla IJT, Poelarends GT, Terpstra T. Bacterial degradation of xenobiotic compounds: evolution and distribution of novel enzyme activity. Environment Microbiol. 2005;7:1868-82.

\section{Submit your next manuscript to BioMed Central and we will help you at every step:}

- We accept pre-submission inquiries

- Our selector tool helps you to find the most relevant journal

- We provide round the clock customer support

- Convenient online submission

- Thorough peer review

- Inclusion in PubMed and all major indexing services

- Maximum visibility for your research

Submit your manuscript at www.biomedcentral.com/submit 